\title{
Optically variable AGN in the three-year VST survey of the COSMOS field $\star, \star \star$
}

 \\ G. Longo ${ }^{3}$, A. Grado ${ }^{5}$, F. Ragosta ${ }^{3,5}$, M. T. Botticella ${ }^{5}$, G. Pignata ${ }^{12,1}$, M. Vaccari ${ }^{13,14}$, M. Radovich ${ }^{15}$, M. Salvato ${ }^{16}$, \\ G. Covone ${ }^{3,4}$, N. R. Napolitano ${ }^{17}$, L. Marchetti ${ }^{18,13,14}$, and P. Schipani ${ }^{5}$
}

1 Millennium Institute of Astrophysics (MAS), Nuncio Monseñor Sotero Sanz 100, Providencia, Santiago, Chile e-mail: demetradecicco@gmail.com; ddecicco@astro.puc.cl

2 Instituto de Astrofísica, Pontificia Universidad Católica de Chile, Av. Vicuña Mackenna 4860, 7820436 Macul, Santiago, Chile

3 Department of Physics, University of Napoli "Federico II", Via Cinthia 9, 80126 Napoli, Italy

${ }^{4}$ INFN - Sezione di Napoli, Via Cinthia 9, 80126 Napoli, Italy

5 INAF - Osservatorio Astronomico di Capodimonte, Via Moiariello 16, 80131 Napoli, Italy

${ }^{6}$ KTH Royal Institute of Technology, Brinellvägen 8, 11428 Stockholm, Sweden

7 Institut für Astro- und Teilchenphysik, Universität Innsbruck, Technikerstraße 25/8, Innsbruck 6020, Austria

${ }^{8}$ Department of Astronomy and Astrophysics, The Pennsylvania State University, University Park, PA 16802, USA

9 Institute for Gravitation and the Cosmos, The Pennsylvania State University, University Park, PA 16802, USA

10 Space Science Institute, 4750 Walnut Street, Suite 2015, Boulder, CO 80301, USA

11 Department of Physics, University of Roma "Tor Vergata", Via della Ricerca Scientifica 1, 00133 Roma, Italy

12 Departamento de Ciencias Fisicas, Universidad Andres Bello, Avda. Republica 252, Santiago, Chile

13 Department of Physics and Astronomy, University of the Western Cape, Private Bag X17, 7535 Bellville, Cape Town, South Africa

14 INAF - Istituto di Radioastronomia, Via Gobetti 101, 40129 Bologna, Italy

15 INAF - Osservatorio Astronomico di Padova, Vicolo dell'Osservatorio 5, 35122 Padova, Italy

16 Max Planck Institut für Extraterrestrische Physik, Giessenbachstraße 1, 85748 Garching bei München, Germany

17 School of Physics and Astronomy, Sun Yat-sen University, Zhuhai Campus, Guangzhou 519082, PR China

18 Department of Astronomy, University of Cape Town, Private Bag X3, Rondebosch 7701, Cape Town, South Africa

Received 10 April 2019 / Accepted 22 May 2019

\begin{abstract}
Context. The analysis of the variability of active galactic nuclei (AGN) at different wavelengths and the study of possible correlations of different spectral windows are a current main field of inquiry. Optical variability has been largely used to identify AGN in multivisit surveys. The strength of a selection based on optical variability lies in the opportunity of analyzing data from surveys of large sky areas by ground-based telescopes. However, the effectiveness of optical variability selection with respect to other multiwavelength techniques has been poorly studied down to the depth that is expected from next-generation surveys.

Aims. Here we present the results of our $r$-band analysis of a sample of 299 optically variable AGN candidates in the VST survey of the COSMOS field, counting 54 visits spread over three observing seasons spanning more than three years. This dataset is more than three times larger than the dataset presented in our previous analysis, and the observing baseline is about eight times longer.

Methods. We push toward deeper magnitudes $(r(\mathrm{AB}) \sim 23.5 \mathrm{mag})$ than were reached in past studies. We made wide use of ancillary multiwavelength catalogs in order to confirm the nature of our AGN candidates, and constrained the accuracy of the method based on spectroscopic and photometric diagnostics. We also performed tests aimed at assessing the relevance of dense sampling in view of future wide-field surveys.

Results. We demonstrate that the method allows the selection of high-purity ( $>86 \%)$ samples. We take advantage of the longer observing baseline to achieve great improvement in the completeness of our sample with respect to X-ray and spectroscopically confirmed samples of AGN (59\% vs. 15\% in our previous work), as well as in the completeness of unobscured and obscured AGN. The effectiveness of the method confirms the importance of developing future more refined techniques for the automated analysis of larger datasets.
\end{abstract}

Key words. galaxies: active - quasars: general - X-rays: galaxies - surveys

\section{Introduction}

Supermassive black holes (SMBHs) are considered ubiquitous guests in the centers of massive galaxies (e.g., Magorrian et al. 1998; Ferrarese \& Merritt 2000). At some point in their lives,

* Table 3 is only available at the CDS via anonymous ftp to cdsarc.u-strasbg.fr (130.79.128.5) or via http://cdsarc. u-strasbg.fr/viz-bin/qcat?J/A+A/627/A33.

$\star \star$ Observations were provided by the ESO programs 088.D-4013, 092.D-0370, and 094.D-0417 (PI G. Pignata). they are powered by accreting matter infall; galaxies experiencing such a phase are known as active galactic nuclei (AGN).

The AGN emission is broadband and covers most of the electromagnetic spectrum, as a result of several continuum emission processes plus emission-line features. These features are typically broader and much more prominent than those that are commonly observed in the spectra of inactive galaxies.

Variability is a signature of AGN emission and is observed at all frequencies, spanning more than 20 orders of magnitude 
(e.g., Padovani et al. 2017, and references therein). It affects both continuum and line emission to an extent that depends on a number of factors: observed spectral range, timescale, presence of radio jets, source luminosity, mass, accretion rate, etc. (e.g., Paolillo et al. 2017). Variations are irregular and aperiodic; their typical timescales range from hours to years, although variations can occasionally be detected on timescales down to minutes or up to $10^{4}$ years (see, e.g., voorwerpjes, Lintott et al. 2009). Several works over the past decades have widely investigated AGN variability in different wavebands, together with the correlations between variations in different spectral windows (e.g., Ulrich et al. 1997; Gaskell \& Klimek 2003). An accurate characterization is possible today thanks to surveys with repeated observations over years or even decades. In particular, multivisit surveys have been extensively used to search for unobscured AGN (e.g., Mushotzky et al. 2004; Klesman \& Sarajedini 2007; Trevese et al. 2008; Schmidt et al. 2010; Villforth et al. 2010; MacLeod et al. 2011; Sarajedini et al. 2011; Choi et al. 2014; Graham et al. 2014, and references therein). These surveys are generally characterized by irregular sampling, with observing gaps due to observational constraints. The features of the observed variability depend on several parameters, such as baseline, number of visits, observing cadence, chosen band, depth, and photometric accuracy.

The next years will see the advent of new-generation telescopes, which are designed to survey wide sky areas with a high cadence. The most highly anticipated of these telescopes is undoubtedly the Large Synoptic Survey Telescope (LSST; see, e.g., LSST Science Collaboration 2009). LSST surveys are designed to cover about half the sky. At completion, they will be 10-100 times deeper than any current wide-field survey, with hundreds to thousands of time samples. About $90 \%$ of the LSST time will be dedicated to the main survey; the remaining $10 \%$ will go into several mini-surveys. These will include ultra-deep observations of 5-10 so-called deep drilling fields (DDFs). The DDFs will target previously surveyed areas, such as COSMOS and the Chandra Deep Field-South (CDFS). For them we can expect up to $\sim 14000$ visits, allowing us to reach co-added depths of ugri $\sim 28.5, z \sim 28$, and $y \sim 27.5 \mathrm{mag}$ over the ten-year survey program. This, combined with the multiwavelength coverage from current and future telescopes, makes the DDFs ideal for AGN studies (see, e.g., Brandt et al. 2018). The first formal survey data from the LSST main survey are expected no sooner than 2023 (best-case scenario). In anticipation, the astronomical community can and must prepare to deal with them, developing fast and reliable classification tools that will be needed to adequately face unprecedented data streams. In this context, we are putting effort into the development and refinement of an effective technique for AGN selection that is based on their optical variability, with the aim of applying it to wider datasets from surveys to come. We elaborated and tested our selection method by analyzing data from the SUpernova Diversity And Rate Evolution (SUDARE; Botticella et al. 2013) survey by the VLT Survey Telescope (VST; Capaccioli \& Schipani 2011). SUDARE is a project that aims at analyzing the trend of the rates of different supernova ( $\mathrm{SN}$ ) types in the redshift range $0.3<z<0.8$. In addition, it investigates possible correlations with the properties of the host galaxies, and any dependence of such correlations on redshift and/or on the stellar population the $\mathrm{SNe}$ belong to. The survey concerns two distinct well-known sky areas: the above-mentioned COSMOS field and CDFS. Both regions have been widely surveyed by a large contingent of ground- and space-based observatories: this makes them an ideal testing ground, given the wealth of multiwavelength data, from
X-rays to radio, that are available for the validation of the method.

The SUDARE survey covers 1 square degree for COSMOS and 4 square degrees for the CDFS. Observations started in late 2011 and are available in the $g, r$, and $i$ bands. De Cicco et al. (2015) published the results of the analysis of the first five months of observations (27 visits) for the COSMOS field. Soon after, Falocco et al. (2015) presented a similar analysis of the first 2 square degrees available for the CDFS: in this case, we had 27 visits for one square degree and 22 visits for the other one, spanning five and three months, respectively. We are currently investigating the other half of the surveyed CDFS area and will present the corresponding results in Poulain et al. (in prep.).

The present paper is dedicated to the analysis of the VST-COSMOS data. The COSMOS campaign has now been extended to a baseline longer than three years, which doubles the number of observing visits. Here we exploit the longer baseline and increased number of observations, in order to verify the predictions of our previous works and increase the number of detected AGN. Moreover, we better constrain the effectiveness of the variability selection with respect to other methods in order to predict the performance of future surveys. We retrieve a larger sample of optically variable AGN candidates due to their typical red-noise variability, thus making progress toward a more complete and effective census of the AGN population in the field.

The present analysis, as well as all of our works published so far, focuses on $r$-band data for both fields. Additional works investigating the other bands, and a combination of all of them, are currently in preparation (see Sect. 5).

The paper is organized as follows: in Sect. 2 we describe our dataset; Sect. 3 illustrates the steps we took to obtain source catalogs from the various visits, and the reduction process leading to the selection of a robust sample of optically variable AGN candidates. Section 4 describes the multiwavelength properties of the selected sample and the various diagnostics we used to validate the nature of our AGN candidates. In Sect. 5 we gather together our results and discuss our findings, also comparing them to those in De Cicco et al. (2015).

\section{VST-COSMOS data}

The VST, located at Cerro Paranal Observatory, is a $2.65 \mathrm{~m}$ optical telescope with a $0.938 \mathrm{~m}$ diameter secondary mirror, a modified Ritchey-Chrétien configuration, and an alt-azimuth mount. Its detector, OmegaCAM (Kuijken 2011), is a mosaic of 32 CCDs, corresponding to a total of 268 million $15 \mu \mathrm{m}$ pixels over a $26 \times 26 \mathrm{~cm}^{2}$ area. The focal plane scale is $0^{\prime \prime} .214 /$ pixel, and the corresponding field of view (FoV) is $1^{\circ} \times 1^{\circ}$. The VSTCOSMOS observations consist of three observing seasons (hereafter, seasons), covering the span from December 2011 to March 2015 , including the data from the season that has been studied in De Cicco et al. (2015).

We distinguish throughout between visits and exposures, a visit being the combination of a number of exposures corresponding to the same observing block (OB). Each visit ${ }^{1}$ consists of several (usually five, but see Table 1 for details) dithered exposures corresponding to individual $1^{\circ} \times 1^{\circ}$ pointings. Exposure reduction and combination were performed by means of the VST-Tube pipeline (Grado et al. 2012), which was designed to process VST data. An overview of the processing

\footnotetext{
1 We note that De Cicco et al. (2015) used the word "epoch" instead of "visit".
} 
steps is reported in De Cicco et al. (2015), while a more exhaustive description can be found in Capaccioli et al. (2015). Essentially, the pipeline takes care of overscan correction, bias subtraction, and flat-field correction, together with CCD gain harmonization and illumination correction; astrometric correction and photometric calibration follow, then single exposures are combined together into visits.

The full dataset originally consisted of 65 visits, but we excluded 11 visits from the present analysis, as detailed below. Information about the remaining 54 visits, which constitute our final dataset, is reported in Table 1 . The first 26 visits in the table cover a five-month baseline and were used for our previous work, described in De Cicco et al. (2015).

A weight map corresponding to each visit accounts for the different noise level of each pixel by associating them a weight, defined as the reciprocal of the pixel variance. As reference, we used the same deep stacked image as in De Cicco et al. (2015), produced as the median of all the exposures having a seeing full width at half-maximum $(F W H M)<0$ '! 80; the corresponding exposure time is $19800 \mathrm{~s}$, and the limiting magnitude is $r(\mathrm{AB}) \approx 26 \mathrm{mag}$ at $\sim 5 \sigma$ above the background r.m.s., while single visits are generally characterized by $r(\mathrm{AB}) \lesssim 24.6 \mathrm{mag}$ for point sources, at the same confidence level. We note that the depth of the VST images is within one magnitude of what LSST is expected to deliver $(r \approx 24.7 \mathrm{mag}$ for a single DDF visit; see Brandt et al. 2018). Although produced only from the first five months of our observing campaign, this stack is obviously deeper than any of our individual visits, and it is sufficient for use as a static reference image.

We here focus on $r$-band data only, which have a three-day observing cadence, while for the $g$ and $i$ bands, the cadence is about ten days; in each case there are several gaps, depending on a number of observational constraints. In the following, magnitudes are quoted in the AB system unless otherwise stated.

\section{Selection of variable sources}

As a first step, we visually inspected each visit in our dataset in order to verify its quality: this led to the exclusion of three visits because of severe aesthetic artifacts and defects. The defects in one of the three visits are mainly arcs originating from reflections internal to the telescope, which are due to the presence of nearby bright stars, while the other two excluded visits are characterized by unusually high noise: this was caused by a very strong and inhomogeneous illumination, originating from moonlight reflections by clouds and some tracking problems with the telescope. The method we adopted to identify optically variable sources, and hence define our sample of AGN candidates, follows the approach proposed by Trevese et al. (2008).

\subsection{Catalog production and aperture selection}

A catalog of sources was obtained from each visit making use of SExtractor (Bertin \& Arnouts 1996): this contains a number of parameters for each object, including positional coordinates, half-light radii, and magnitudes through a set of fixed apertures. We fed these weight maps to SExtractor so that in the process of catalog extraction, the different quality of each pixel in each visit is taken into account.

Identifying AGN requires an aperture size that allows collecting the bulk of the flux from the nucleus of a galaxy while minimizing the contribution from the host galaxy itself and possible nearby sources. De Cicco et al. (2015) measured the source flux within a $2^{\prime \prime}$ diameter, which typically encloses $\approx 70 \%$
Table 1. COSMOS dataset.

\begin{tabular}{|c|c|c|c|}
\hline Visit & OB-ID & obs. date & $\begin{array}{c}\text { Seeing }(F W H M) \\
(\operatorname{arcsec})\end{array}$ \\
\hline 1 & 611279 & 2011-Dec-18 & 0.64 \\
\hline 2 & 611283 & 2011-Dec-22 & 0.94 \\
\hline 3 & 611287 & 2011-Dec-27 & 1.04 \\
\hline 4 & 611291 & 2011-Dec-31 & 1.15 \\
\hline 5 & 611295 & 2012-Jan-02 & 0.67 \\
\hline 6 & 611299 & 2012-Jan-06 & 0.58 \\
\hline 7 & 611311 & 2012-Jan-18 & 0.62 \\
\hline 8 & 611315 & 2012-Jan-20 & 0.88 \\
\hline 9 & 611319 & 2012-Jan-22 & 0.81 \\
\hline 10 & 611323 & 2012-Jan-24 & 0.67 \\
\hline 11 & 611327 & 2012-Jan-27 & 0.98 \\
\hline 12 & 611331 & 2012-Jan-29 & 0.86 \\
\hline 13 & 611335 & 2012-Feb-02 & 0.86 \\
\hline 14 & 611351 & 2012-Feb-16 & 0.50 \\
\hline 15 & 611355 & 2012-Feb-19 & 0.99 \\
\hline 16 & 611359 & 2012-Feb-21 & 0.79 \\
\hline 17 & 611363 & 2012-Feb-23 & 0.73 \\
\hline 18 & 611367 & 2012-Feb-26 & 0.83 \\
\hline 19 & 611371 & 2012-Feb-29 & 0.90 \\
\hline 20 & 611375 & 2012-Mar-03 & 0.97 \\
\hline 21 & 611387 & 2012-Mar-13 & 0.70 \\
\hline 22 & 611391 & 2012-Mar-15 & 1.08 \\
\hline 23 & 611395 & 2012-Mar-17 & 0.91 \\
\hline 24 & 768813 & 2012-Мay-08 & 0.74 \\
\hline 25 & 768817 & 2012-May-11 & 0.85 \\
\hline 26 & 768820 & 2012-May-17 & 0.77 \\
\hline 28 & 986611 & 2013-Dec-27 & 0.72 \\
\hline 29 & 986614 & 2013-Dec-30 & 1.00 \\
\hline 30 & 986617 & 2014-Jan-03 & 0.86 \\
\hline 31 & 986620 & 2014-Jan-05 & 0.81 \\
\hline 32 & 986626 & 2014-Jan-12 & 0.73 \\
\hline 33 & 986630 & 2014-Jan-21 & 1.18 \\
\hline 34 & 986633 & 2014-Jan-24 & 0.80 \\
\hline 37 & 986648 & 2014-Feb-09 & 1.28 \\
\hline 38 & 986652 & 2014-Feb-19 & 0.89 \\
\hline 39 & 986655 & 2014-Feb-21 & 0.93 \\
\hline 40 & 986658 & 2014-Feb-23 & 0.81 \\
\hline 41 & 986661 & 2014-Feb-26 & 0.81 \\
\hline 42 & 986664 & 2014-Feb-28 & 0.77 \\
\hline 44 & 986670 & 2014-Mar-08 & 0.91 \\
\hline 45 & 986674 & 2014-Mar-21 & 0.96 \\
\hline 46 & 986677 & 2014-Mar-23 & 0.92 \\
\hline 47 & 986680 & 2014-Mar-25 & 0.66 \\
\hline 48 & 1095777 & 2014-Mar-29 & 0.89 \\
\hline 49 & 1095783 & 2014-Apr-04 & 0.58 \\
\hline 50 & 986683 & 2014-Apr-07 & 0.61 \\
\hline 51 & 1136410 & 2014-Dec-03 & 1.00 \\
\hline 53 & 1136457 & 2015-Jan-10 & 0.71 \\
\hline 55 & 1136481 & 2015-Jan-28 & 0.90 \\
\hline 56 & 1136490 & 2015-Jan-31 & 0.73 \\
\hline 57 & 1136503 & 2015-Feb-15 & 0.70 \\
\hline 60 & 1136531 & 2015-Mar-10 & 0.80 \\
\hline 61 & 1136540 & 2015-Mar-14 & 0.84 \\
\hline 62 & 1136543 & 2015-Mar-19 & 1.00 \\
\hline Stacked & - & - & 0.67 \\
\hline
\end{tabular}

Notes. Visit number, OB identification number, date, and seeing FWHM for the 54 visits we used for this analysis. Visits are listed in chronological order, and the sequence of their IDs in the first column lacks some numbers because these correspond to the eight visits that we excluded from the analysis (see Sect. 3.1). Visit 53 was obtained by the combination of ten exposures, for a total exposure time of $3600 \mathrm{~s}$; for all the remaining visits, five exposures were combined together, and the total exposure time is $1800 \mathrm{~s}$.

of the flux from a point-like object. In order to take into account the effect of seeing, we then computed corrective factors for each visit, making use of growth curves of reference stars. A growth 




Fig. 1. Magnitude difference between visits 3 and 49 . Visit 49 is used as a reference. Although all the sources with an available measurement of their magnitude are shown, the sources we used to determine the aperture corrective factors have $r(\mathrm{AB})$ magnitudes in the range 16-21. Dark and light gray dots represent magnitudes before and after the correction, respectively; similarly, the dashed and solid lines correspond to the average magnitude difference before and after the correction, respectively. The first defines the corrective factor, which in this case is $\approx 0.22$. The obtained value varies depending on the visit seeing; it is higher for higher seeing values. Visit 3 has a seeing value of $1{ }^{\prime \prime} 04$, which is one of the highest in our set of visits.

curve shows how the fraction of flux that is collected from a source changes as a function of the aperture size ${ }^{2}$.

Here we adopt a different approach, following Trevese et al. (2008), which essentially consists of normalizing all visits to a reference visit. We chose visit 49 , which has the second-best seeing (see Table 1) and is free from significant aesthetic artifacts (while visit 14, which is the one with the best seeing, is affected by bad pixels and artifacts). For each visit, we then selected all the sources with $2^{\prime \prime}$ diameter aperture $r$ magnitudes in the range 16-21 (to avoid very bright and saturated or faint and noisy objects) and computed the average magnitude difference $\langle\Delta \mathrm{mag}\rangle$ with respect to the reference visit. The catalogs of sources obtained from different visits were matched by requiring the distance between positional coordinates to be $\leq 1^{\prime \prime}$. We subtracted from all $2^{\prime \prime}$ aperture magnitudes in each visit the corresponding corrective factor in order to account for seeing and calibration differences. The magnitude difference between visits 3

2 In De Cicco et al. (2015) for each visit we determined a corrective factor: we defined it as the ratio of the flux from the reference star enclosed in a 2 "diameter aperture to the flux corresponding to $90 \%$ of the total. In this way, independent of the visit seeing, each corrected magnitude corresponds to $90 \%$ of the flux collected in the chosen aperture. We note that the $90 \%$ choice is arbitrary: what matters is that the fraction of collected flux be the same for each source in each visit. The reference stars we chose were detected in each visit and were not saturated; they did not have close neighbors, and were distant from possibly defected regions of the image (see next section).

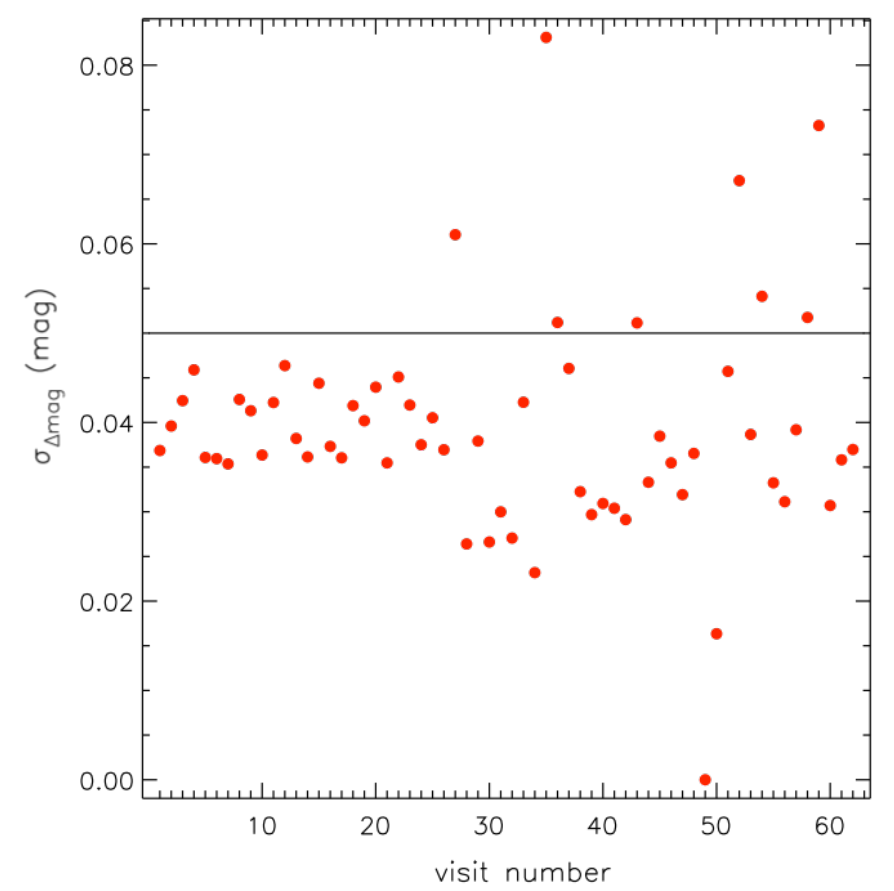

Fig. 2. $\sigma_{\Delta \mathrm{mag}}$ for each visit. The sources we used to determine the aperture corrective factors have $r(\mathrm{AB})$ magnitudes in the range 16-21. The points above the black line correspond to the eight visits that we excluded from our dataset.

and 49 for all the sources in the field before and after the correction is shown in Fig. 1 as an example.

As a further step, we computed the r.m.s. deviation $\sigma_{\Delta \mathrm{mag}}$ of $\Delta$ mag for each visit in order to quantify the calibration uncertainty with respect to the reference visit. $\sigma_{\Delta \mathrm{mag}}$ for each visit is reported in Fig. 2. We decided to exclude from our dataset the visits characterized by $\sigma_{\Delta \text { mag }}$ values higher than 0.05 ; these correspond to eight visits that are represented by the most scattered points in the figure. Scattered points corresponding to low values are not to be taken into account because their photometry is the closest to the one in the reference visit. The adopted limit is arbitrary, and some excluded visits are indeed very close to the threshold. We tested how the inclusion of these four visits would affect the analysis. Although including them would add a few $(<10)$ more sources to the sample of optically variable AGN candidates, it would also introduce several dozen contaminants. This means that although excluding these visits may be considered a conservative approach, excluding them did not significantly affect our results.

\subsection{Defects and masks}

Observations of the COSMOS field from the first season correspond to the very beginning of VST activity, and various early visits turned out to be affected by a number of defects in the detector electronics, as well as aesthetic artifacts. VST datausers, including us, had to address them for the first time, and the most natural path to follow resulted in a conservative approach. Some of the problems arose from a poor knowledge of the detector response and were fixed in the following months. In particular, the most relevant problem was a CCD characterized by random variations in its gain factor, which resulted in a high concentration of fake sources in the corresponding area of the detector. This led to the exclusion of the image region corresponding to the CCD from the affected visits. Similarly, other regions were 
excluded in some or all of the visits, depending on the circumstances. Examples are the regions at the edge of each visit (corresponding to $\mathrm{a} \approx 2^{\prime}-4^{\prime}$ width each side), characterized by a very low signal-to-noise ratio $(\mathrm{S} / \mathrm{N})$, satellite tracks, regions affected by reflections of scattered light, and last but not least, bright stellar halos. The last ones are a common feature in wide-field surveys and constitute the main problem that we had to address by applying suitable masks to the images at issue. Bright stellar halos and spikes were masked making use of the regions produced by the Pulecenella code developed by Huang et al. (2011). These initial masks were improved upon by adding, when necessary, additional eye-selected regions to be excluded for each visit.

Specifically, for one individual visit, an additional region affected by reflections of scattered light was masked. Satellite tracks were not masked, but the potential problems they could cause were minimized by resorting to a sigma-clipping algorithm (see Sect. 3.3). The masking process reduced the number of objects in each catalog by some tens of thousands, representing $20 \%-25 \%$ of the whole source catalog, depending on the seeing value (higher seeing required larger masked areas).

\subsection{Sample of AGN candidates}

We cross-matched the catalogs of sources obtained for each visit by matching positional coordinates within a $1^{\prime \prime}$ radius, in order to obtain the light curves of all the detected objects. Source separations are $<0^{\prime \prime} .26$ in $95 \%$ of the cases. The master catalog thus obtained includes all the variable and non-variable sources detected in at least two visits and with an average magnitude $r \leq 23.5 \mathrm{mag}$. Our previous work focused on sources with a $r \leq 23 \mathrm{mag}$; here we extend the sample down to fainter magnitudes, consistent with the single-visit completeness limit.

The master catalog contains 25452 sources, including a number of objects that were detected in just a few visits. Many of these are either fast transients and/or more likely spurious sources. In order to minimize contamination from such objects, we made a further cut and required the sources in our final cata$\log$ to be detected in at least half the visits.

We note that we adopted a different criterion than in De Cicco et al. (2015), where we required sources to be detected in at least $20 \%$ of the visits. The minimum number of visits is somewhat arbitrary because it depends on the type of source to detect or exclude and its variability. Here we increased the minimum visit requirement based on our experience with the first five months of data, and considering the longer time span of our light curves (which increases the likelihood of detecting variability from fainter AGN). We find that in any case, $90 \%$ of the sources in our master catalog are detected in at least half the visits. This percentage would rise to $99 \%$ if we required sources to be detected in $20 \%$ of the visits, but the sample of variable AGN candidates would be almost the same as the sample we obtain with the adopted threshold, with some dozen additional sources that would be mostly contaminants. We therefore opted for the more conservative threshold.

The number of visits in each light curve also affects the detection significance for variable sources through the different number of degrees of freedom in each light curve (see discussion in De Cicco et al. 2015). To assess the relevance of this effect, we performed simulations including each time a different number of visits, and we found that the detection threshold for variable sources differs by less than $15 \%$ between sources detected in all visits and those detected in just half of them.

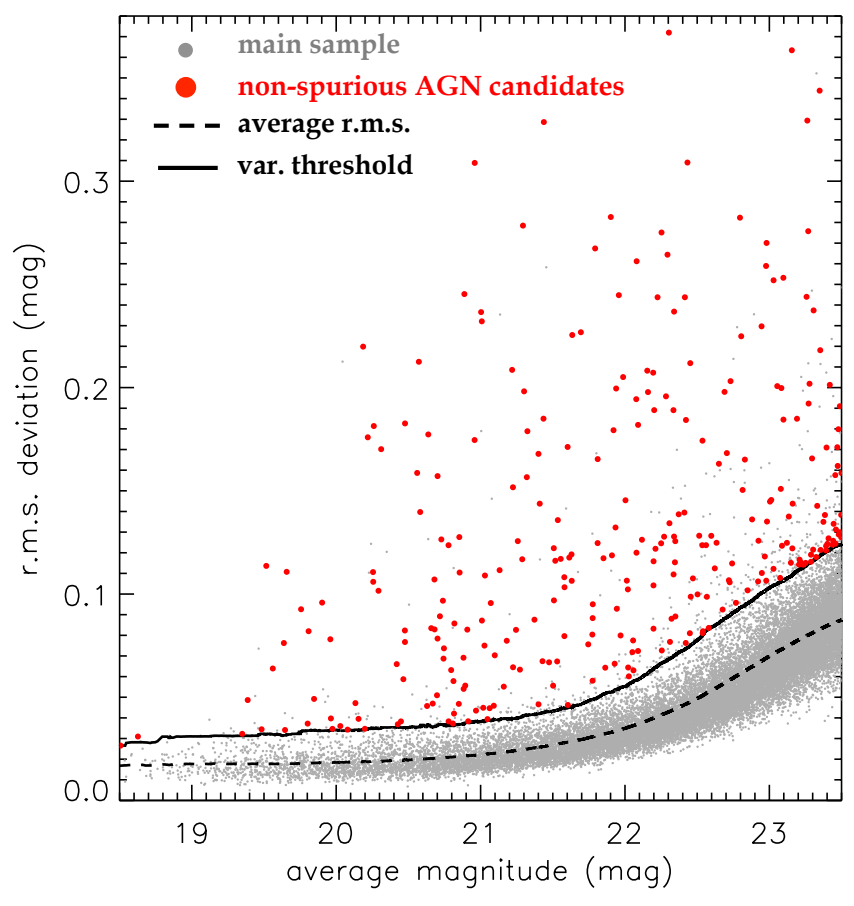

Fig. 3. Light curve r.m.s. $\sigma^{l c}$ as a function of the average magnitude $\left\langle\operatorname{mag}^{l c}\right\rangle$ for all the sources in the main sample (small gray dots). The dashed line represents the running average of the r.m.s., while the solid line defines the variability threshold. Objects above the threshold are considered to be variable, and large red dots identify non-spurious AGN candidates (flag 1 or 2 , see further in the text).

The applied selection criteria returned a sample of 22927 sources (hereafter, the main sample). We point out that this includes both variable and non-variable sources.

In order to define a variability threshold, we computed the average magnitude $\left\langle\operatorname{mag}_{i}^{l c}\right\rangle$ and the corresponding r.m.s. deviation $\sigma_{i}^{l c}$ for each source $i$ from the corresponding light curve:

$\left\langle\operatorname{mag}_{i}^{l c}\right\rangle=\frac{1}{N_{\mathrm{vis}}} \sum_{j=1}^{N_{\mathrm{vis}}} \operatorname{mag}_{i}^{j}, \quad \sigma_{i}^{l c}=\left[\frac{1}{N_{\mathrm{vis}}} \sum_{j=1}^{N_{\mathrm{vis}}}\left(\operatorname{mag}_{i}^{j}-\left\langle\operatorname{mag}_{i}^{l c}\right\rangle\right)^{2}\right]^{\frac{1}{2}}$,

where the superscript $l c$ stands for "light curve", $j$ runs through the visits, and $N_{\text {vis }}$ is the number of visits where we detect the source $i$. Both $\left\langle\operatorname{mag}^{l c}\right\rangle$ and $\sigma^{l c}$ were obtained through a sigmaclipping algorithm, rejecting $>5 \sigma$ outliers, in order to minimize spurious contributions to magnitude variations that are due, for instance, to residual aesthetic defects (e.g., satellite tracks, cosmic rays, and stellar diffraction spikes).

After we measured the properties of all light curves in our main sample, we extracted the sample of variable sources. We initially defined as variable candidates all sources exhibiting a $\sigma^{l c}$ in excess of the 95th percentile of the $\sigma^{l c}$ distribution, over a running $0.5 \mathrm{mag}$ wide bin centered on the magnitude of each source. We identified 482 sources (2.1\% of the main sample) above the variability threshold, which constitute our preliminary sample of AGN candidates. Figure 3 shows the variability threshold and the sample of AGN candidates in the plane of $\sigma^{l c}$ versus $\left\langle\mathrm{mag}^{l c}\right\rangle$.

We expect the variability of some sources in our preliminary sample to be spurious due to a number of factors: the irregular morphology of some galaxies, which makes it difficult to identify the source centroid in the visits with the highest seeing values; problematic detector areas (e.g., hot pixels) or noise-dominated 
regions (e.g., the edges of a frame) where sources happen to fall; contamination from a close companion. In principle, a change in the input extraction parameters could help reduce such occurrences, but in any case, when a source has a very close companion, and in particular, when this object is very bright, it is not possible to determine whether variability is an intrinsic property of the source or arises from visit-to-visit point spread function (PSF) variations combined with contamination from the companion. We also expect our sample to include a number of contaminants, such as variable stars and transient events (e.g., SNe). The identification and analysis of SNe in our dataset is currently ongoing (Ragosta et al., in prep.); De Cicco et al. (2015) measured a $14 \%$ contamination rate due to $\mathrm{SNe}$ in our final sample of variable sources. The contamination is defined as the number of confirmed non-AGN divided by the number of AGN candidates; it can also be computed for specific classes of sources (e.g., SNe, stars) when information about them is available.

Our preliminary sample of optically variable sources includes 90 objects that suffer from blending with neighboring galaxy at least in the visits with poor seeing. We inspected the snapshots of each source in each visit, and they revealed that the centroid position can vary substantially, migrating from one object to the other, depending on the visit. This means that in either case, the corresponding measured flux is possibly incorrect, therefore we excluded them from our analysis. This left 392 candidates. In order to identify and reject spurious sources and contaminants, we visually inspected the snapshots of each source in each visit, and flagged sources with a quality label ranging from 1 to 3 on the basis of the following guidelines:

1. strong candidate; no detected problems or defects (232 sources);

2. likely variable candidate; neighbor might affect the source, or minor problems (67 sources);

3. very doubtful variability; likely spurious (93 sources).

Sources are flagged as 3 either because of their irregular shape (43 objects), which does not allow reliable identification of a center, or because of a nearby saturated object (50 objects); in this case, we used the same empirical selection criterion that we set in our previous work, and required the centroid-to-centroid distance to be $\leq 2^{\prime \prime}$ and the magnitude difference of the two sources to be $<1.5 \mathrm{mag}$ for a source to be a flag 3 because we assumed that in such a case, the light from one source strongly affects the other.

We point out that the sample cleaning process can be automated in part using SExtractor flags and other diagnostics to a priori exclude sources with close neighbors, those with cross-contamination, and so on. In this case, we resorted to a manual approach because we had only a limited number of candidates to inspect and because we gained in this way a reference sample for optimizing the automatic procedure in the future. The implementation is already in progress for the followup work we described above that combines $g, r$, and $i$ light curves, and will be an unavoidable approach for any future larger sample.

In Fig. 4 we present one source per class as an example; images for flags 1 and 2 are from the visit with the second-best seeing, while for flag 3 we show an image from the visit with the poorest seeing in order to show how hard it is to identify the source above the complex background because of the bright extended neighbor. The variability analysis that we describe in the following is limited to sources labeled 1 or 2 (hereafter, the robust sample), consisting of 299 sources and hence constituting $1.3 \%$ of the main sample and $62 \%$ of the initial AGN candidates with $r$ mag $\leq 23.5 \mathrm{mag}$.
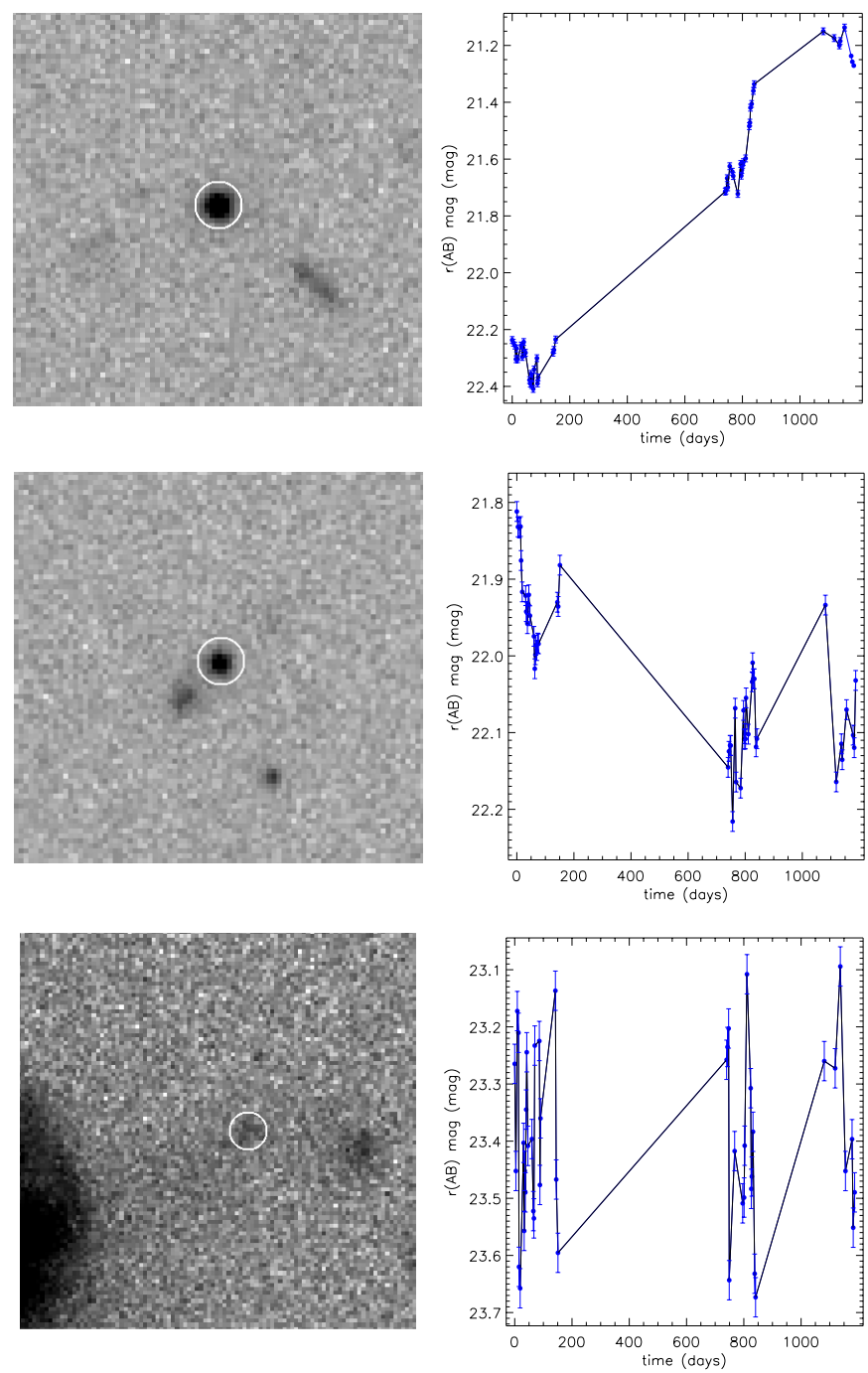

Fig. 4. Examples of variable AGN candidates assigned to different quality classes, with corresponding light curves. The images in the upper and middle panel are from visit 49, which has the second-best seeing; the image in the lower panel is from visit 33, which has the poorest seeing. White circles correspond to the $2^{\prime \prime}$ diameter aperture and are centered on the average object coordinates. Objects labeled 1 (upper panel) are generally isolated and free from aesthetic defects. For objects belonging to class 2 (middle panel), potential problems (e.g., the presence of a neighbor) must be taken into account. Objects labeled 3 (lower panel) are probably spurious variable sources. The case of a source lying in the halo of an extended and bright neighbor (on the far left) is shown as an example: from the corresponding light curve, we can see that its erratic variability, well in excess of the photometric error, is likely due to an incorrect subtraction of the variable background because of the bright neighbor. The error bars for each source are defined as the $95 \%$ uncertainty on the source magnitude.

\section{Validation and characterization of the AGN candidates}

In this section we investigate the nature and properties of the sources in our robust sample, aiming at confirming AGN and at classifying as many of the remaining sources as possible. We intend to assess to what extent optical variability is a reliable indicator of the presence of an AGN, and hence a powerful alternative to other more expensive and/or time-consuming techniques (e.g., X-ray identification) that are usually employed in the search for AGN. 
Because the COSMOS field has been studied frequently and in detail, several catalogs at different wavelengths are available for most of the sources, and classification already exists for part of our sample. This allowed us to validate our results by means of extensive ancillary data.

\subsection{X-ray counterparts}

$\mathrm{X}$-ray emission, especially if coupled with variability, is the strongest and most reliable indicator of the presence of an AGN (e.g., Brandt \& Alexander 2015). We here made use of two Xray catalogs of COSMOS sources to identify possible X-ray counterparts for our main sample:

- The catalog containing optical and near-infrared (NIR) counterparts of the sources in the Chandra-COSMOS Legacy Catalog (Marchesi et al. 2016; Civano et al. 2016), consisting of $4016 \mathrm{X}$-ray emitters over an area of 2.2 square degrees. The catalog is the result of a $4.6 \mathrm{Ms}$ program with an exposure of $\simeq 160 \mathrm{ks}$ in the central 1.5 square degrees and $\simeq 80 \mathrm{ks}$ in the surrounding area. The limiting depth corresponds to fluxes of $2.2 \times 10^{-16} \mathrm{erg} \mathrm{cm}^{-2} \mathrm{~s}^{-1}, 1.5 \times 10^{-15} \mathrm{erg} \mathrm{cm}^{-2} \mathrm{~s}^{-1}$, and $8.9 \times 10^{-16} \mathrm{erg} \mathrm{cm}^{-2} \mathrm{~s}^{-1}$ in the $0.5-2,2-10$, and 5-10 keV bands, respectively. The catalog provides a considerable volume of information, including optical counterparts for 3899 (97\%) of the 4016 sources, spectroscopic classification (broad-line AGN (BLAGN); non-BLAGN; star) for $\approx 42 \%$ of the sample, and photometric classification (obscured/unobscured AGN; galaxy; star) based on the fitting of the spectral energy distribution (SED) for $96 \%$ of the sample.

- The XMM-COSMOS Point-like Source catalog (Brusa et al. 2010), including 1674 X-ray sources with optical counterparts. The corresponding observations $(\simeq 60 \mathrm{ks})$ in this case are shallower and a little less extended (2 square degrees) than the Chandra-COSMOS Legacy Survey; the flux limits in the $0.5-2 \mathrm{keV}, 2-10 \mathrm{keV}$, and 5-10 keV energy bands are $\approx 1.7 \times 10^{-15} \mathrm{erg} \mathrm{cm}^{-2} \mathrm{~s}^{-1}, \approx 9.3 \times 10^{-15} \mathrm{erg} \mathrm{cm}^{-2} \mathrm{~s}^{-1}$, and $\approx 1.3 \times 10^{-14} \mathrm{erg} \mathrm{cm}^{-2} \mathrm{~s}^{-1}$, respectively, over $90 \%$ of the area. Spectroscopic classification (BLAGN; narrow-line AGN (NLAGN); normal or star-forming galaxy) is available for about half the sample, and a best-fit SED template by Salvato et al. (2009) is provided for $97 \%$ of the sample. Although this catalog is far shallower than the Chandra catalog, it provides some useful details about the nature of the sources (see Sect. 4.2).

The two X-ray catalogs provide information for $1815 \mathrm{X}$-ray sources in the VST-COSMOS FoV (excluding masked areas), with coordinates for their optical counterparts reported in the Xray catalogs themselves. Nevertheless, not all of them have a VST counterpart. Specifically, we found:

-719 X-ray sources with magnitude $r(\mathrm{AB})<23.5$ mag and a VST counterpart;

- 575 sources with magnitude $r(\mathrm{AB})>23.5 \mathrm{mag}$, hence not taken into account in what follows. Magnitude values were retrieved either from the COSMOS2015 catalog presented in Laigle et al. (2016) or from the XMM-COSMOS catalog; the latter reports $r(\mathrm{AB})$ magnitudes from Capak et al. (2007) for $98 \%$ of the sources. In only two cases are the magnitudes from the COSMOS Intermediate and Broad Band Photometry catalog;

- 512 sources with a VST counterpart but detected only in a few visits because they are close to the detection limit, and hence excluded from the main sample according to the visit threshold we set (see Sect. 3.3);

-5 sources with $r(\mathrm{AB})<23.5 \mathrm{mag}$ and with a very close, very bright neighbor, which prevented the detection in VST data;
-3 sources for which we find a VST counterpart with a matching radius larger than $1^{\prime \prime}$;

-one source that appears very blurred in the VST images and has no magnitude estimate in either of the two X-ray catalogs.

The sample of X-ray sources that have a VST counterpart and fulfill our selection criteria hence consists of 719 objects (hereafter, the X-ray sample), or $3 \%$ of the 22927 sources in the main sample. The match of the robust sample with the X-ray sample revealed 250 AGN candidates (84\% of the robust sample: hereafter, X-ray emitting variable AGN candidates) with an X-ray counterpart; this implies that 250 out of $719 \mathrm{X}$-ray sources with bright optical counterparts (35\%) appear optically variable in our catalog.

All but one of the $250 \mathrm{X}$-ray emitting variable AGN candidates have an X-ray counterpart in the deeper Chandra catalog, and we thus preferentially quote Chandra values.

The ratio of the X-ray-to-optical flux $(\mathrm{X} / \mathrm{O})$ of a source is traditionally defined as (Maccacaro et al. 1988)

$X / O=\log \left(f_{\mathrm{X}} / f_{\text {opt }}\right)=\log f_{\mathrm{X}}+\frac{\operatorname{mag}_{\text {opt }}}{2.5}+C$,

where $f_{\mathrm{X}}$ is the X-ray flux measured in a chosen energy band, while mag $_{\text {opt }}$ is the optical magnitude at a chosen wavelength, and $C$ is a constant that depends on the magnitude system adopted for the observations. In our case, the X-ray fluxes are from the Chandra catalog and are measured in the $2-10 \mathrm{keV}$ band, while the optical magnitudes are the VST $r(\mathrm{AB})$ magnitudes. This leads to a value for the constant $C=1.0752$. Stars and inactive galaxies typically exhibit $X / O<-2$ (e.g., Mainieri et al. 2002; Xue et al. 2011), while AGN are usually characterized by $-1 \leq X / O \leq 1$, hence the $\mathrm{X} / \mathrm{O}$ constraints on our sources can help unveil their nature. In Fig. 5 we show the hard $(2-10 \mathrm{keV}) \mathrm{X}$-ray flux versus $r$-band magnitude for the sources in the robust sample with an X-ray counterpart. It is apparent that all but six of the X-ray emitting variable AGN candidates lie in the AGN locus, while six sources have an $X / O>1$. Following Civano et al. (2012), we consider all 250 X-ray emitting variable AGN candidates as AGN because of their $\mathrm{X} / \mathrm{O}$ values. This means that if no additional information about our robust sample were available, we could be confident that $84 \%$ (250/299) are indeed AGN on the sole basis of the X/O diagram. The $84 \%$ represent a lower limit on the purity of our sample. We note that the sources out of the AGN locus on this diagram could be AGN as well: indeed, this diagnostic is typically biased against X-ray faint AGN (see, e.g., Salvato et al. 2018). We define the completeness as the ratio of the sources that we confirm as AGN (250 objects) and the number of AGN in our X-ray sample (i.e., the X-ray sources that lie in the AGN region on the $\mathrm{X} / \mathrm{O}$ diagram, i.e., 668 sources). This results in a fraction of $37 \%$ $(250 / 668)$ completeness. We point out that there is always some degree of uncertainty in the definition of the $\mathrm{X} / \mathrm{O}$ of a source because of the intrinsic variability of the source itself combined with the non-simultaneity of the X-ray and optical observations (e.g., Paolillo et al. 2017; Chiaraluce et al. 2018), and the changing contributions from the host galaxy in the optical band as a function of redshift (see, e.g., Fig. 3 of Alexander et al. 2002).

\subsection{Spectroscopic and photometric classification}

In Sect. 4.1 we mentioned that both Chandra and XMM catalogs provide a spectroscopic and a photometric classification for part of the X-ray emitters in each of the catalogs. In the first catalog, objects with a spectroscopic classification are labeled as 


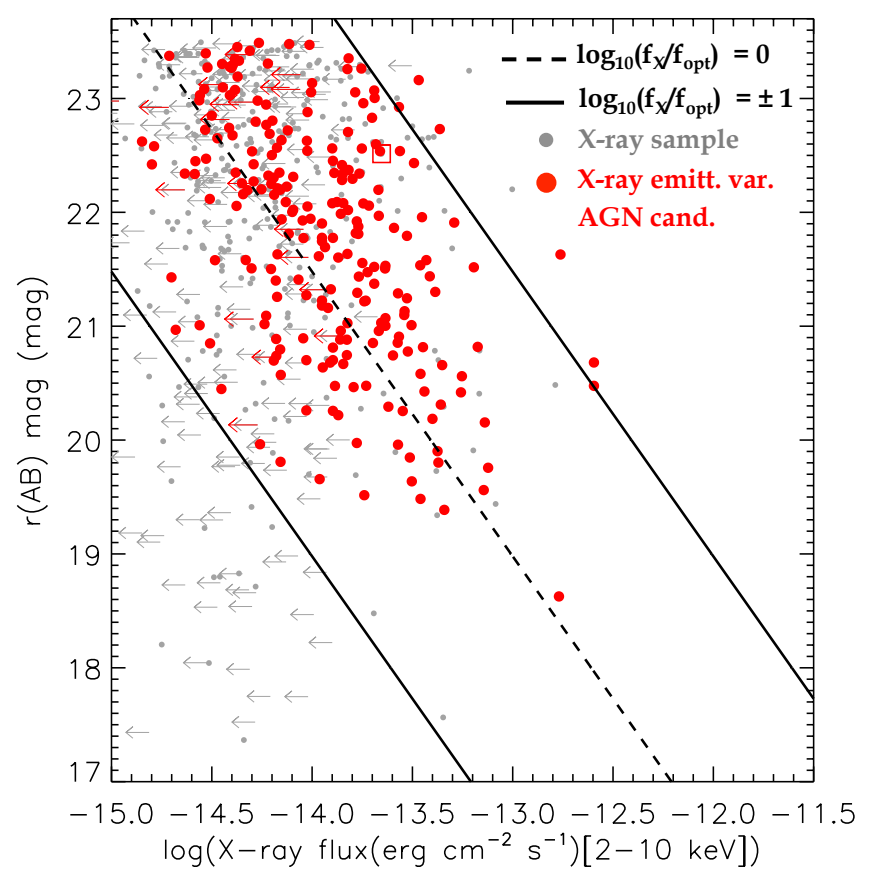

Fig. 5. $r(\mathrm{AB})$ magnitude vs. hard $(2-10 \mathrm{keV}) \mathrm{X}$-ray flux for the 250 $\mathrm{X}$-ray emitting variable AGN candidates (large red symbols denote Chandra detections, and an open box denotes XMM detection). The small gray symbols denote the remainder of the X-ray sample. Leftward arrows indicate that only upper limits of the $\mathrm{X}$-ray flux values are available. The dashed line corresponds to $X / O=0$. The lower and upper solid lines represent $X / O=-1$ and $X / O=1$, respectively, and define the AGN locus.

BLAGN if their spectra show at least one broad $(F W H M>$ $2000 \mathrm{~km} \mathrm{~s}^{-1}$ ) emission line, while non-BLAGN could be NLAGN or star-forming galaxies: this is either because most of the sources at issue are characterized by low $\mathrm{S} / \mathrm{N}$ spectra, or because the waveband in which the spectra are obtained does not allow using optical emission line diagnostics, which would help separating the two classes of objects. The photometric classification is instead derived through best-fit templates of the broadband SEDs, and sources are divided into unobscured AGN, obscured AGN, inactive galaxies, and stars. A cross-match of the two classifications confirmed that $82 \%$ of the BLAGN correspond to unobscured AGN. The match is not higher because BLAGN SEDs, especially for low-luminosity AGN, suffer from stellar light contamination; non-BLAGN are matched to obscured AGN in $23 \%$ of the cases and to galaxies in 74\% of the cases (Marchesi et al. 2016).

The XMM catalog classifies sources as BLAGN, NLAGN, and inactive galaxies. BLAGN must fulfill the same criterion as in the Chandra catalog. Sources flagged as NLAGN typically have spectra that are characterized by unresolved high-ionization emission lines with line ratios suggesting AGN activity, while inactive galaxy spectra are generally consistent with those of starforming or normal galaxies, and have rest-frame hard X-ray luminosity $L_{\mathrm{X}}<2 \times 10^{42} \mathrm{erg} \mathrm{s}^{-1}$ when detected in the hard X-rays. Part of the best-fit SED templates correspond to unobscured (also known as Type 1) and obscured (Type 2) AGN (Brusa et al. 2010).

In the VST X-ray sample (see Sect. 4.1) 600 out of 719 sources have a spectroscopic classification from either of the two catalogs; moreover, all but 6 out of 719 sources have a photometric classification. Among the spectroscopically classified objects, we find 243 unobscured AGN and 140 obscured AGN based on the Chandra catalog and always adopting the spectroscopic over the photometric classification. While the definitions for unobscured AGN are the same in the two X-ray catalogs, the label "non-BLAGN" in the Chandra catalog is ambiguous (see details in Sect. 4.1) and therefore not sufficient to classify a source. The robust sample also includes 11 sources that are spectroscopically classified as stars.

Our 250 X-ray emitting variable AGN candidates are spectroscopically classified as 200 unobscured AGN; 25 obscured AGN; two stars; five sources with uncertain classification, including two possible inactive galaxies; and 18 unclassified sources. Nonetheless, we note that 17 out of the 23 sources that are not classified as obscured/unobscured AGN (18 unclassified +5 uncertain), including the two possible inactive galaxies, have X-ray luminosities $L_{\mathrm{X}}>3 \times 10^{42} \mathrm{erg} \mathrm{s}^{-1}$, indicative of AGN (e.g., Brandt \& Hasinger 2005); moreover, by selection all 23 sources lie on the AGN stripe in the X/O diagram. This means that spectroscopic information alone allows us to identify 225 AGN and two stars. By combining this information with the $\mathrm{X} / \mathrm{O}$ information and taking into account the $\mathrm{X}$-ray luminosities, we can therefore classify as AGN 248 out of the 250 X-ray emitting variable AGN candidates, excluding the two stars, but including the two possible inactive galaxies.

The completeness of the variability selection with respect to the spectroscopically confirmed sample is defined as the ratio of spectroscopically confirmed AGN (in the robust sample) to the expected AGN (in the X-ray sample), and is 59\% (225/383), but if they are computed separately for unobscured and obscured AGN, we obtain $200 / 243=82 \%$ and $25 / 140=18 \%$, respectively. These fractions are much higher than those reported in De Cicco et al. (2015). This is one of the main results of this work and is discussed further in Sect. 5.

\subsection{Color-based classification}

Diagrams comparing source colors are widely used to distinguish different classes of objects (see, e.g., Boutsia et al. 2009; Nakos et al. 2009), based on the fact that sources tend to have distinct SEDs and occupy distinct loci on such diagrams, depending on their nature. In order to obtain color diagrams, we made use of data from two additional COSMOS catalogs:

- The COSMOS2015 catalog (Laigle et al. 2016), which contains photometry and physical parameters for more than half a million objects in several filters (including intermediate- and narrow-band objects) spanning a wide range of wavelengths. It also provides matches with optical, X-Ray, UV, IR, and radio catalogs, as well as previous versions of the COSMOS multiband catalog. We use this catalog to obtain the magnitudes we need in the $r, z$, and $k$ bands, down to a magnitude $r(\mathrm{AB}) \approx 28 \mathrm{mag}$. As stated in Sect. 3.3, we limit our analysis to sources brighter than $23.5 r(\mathrm{AB}) \mathrm{mag}$. We report the magnitude limit of the COSMOS2015 catalog only to show that the magnitude range we investigate here is fully covered.

- The COSMOS ACS catalog (Koekemoer et al. 2007; Scoville et al. 2007) from the Hubble Space Telescope (HST), obtained from 575 pointings of the Advanced Camera for Surveys (ACS). This catalog is mainly used because it provides a morphological classification based on the SExtractor stellarity index, ranging from 0 (extended source) to 1 (point-like source). Given the exquisite HST resolution, this catalog allows us to better distinguish galaxies from stars and quasars, as discussed below.

\subsubsection{Optical-NIR diagnostic}

Nakos et al. (2009) showed that the use of the $r-z$ versus $z-K$ diagram is very effective in distinguishing stars from 


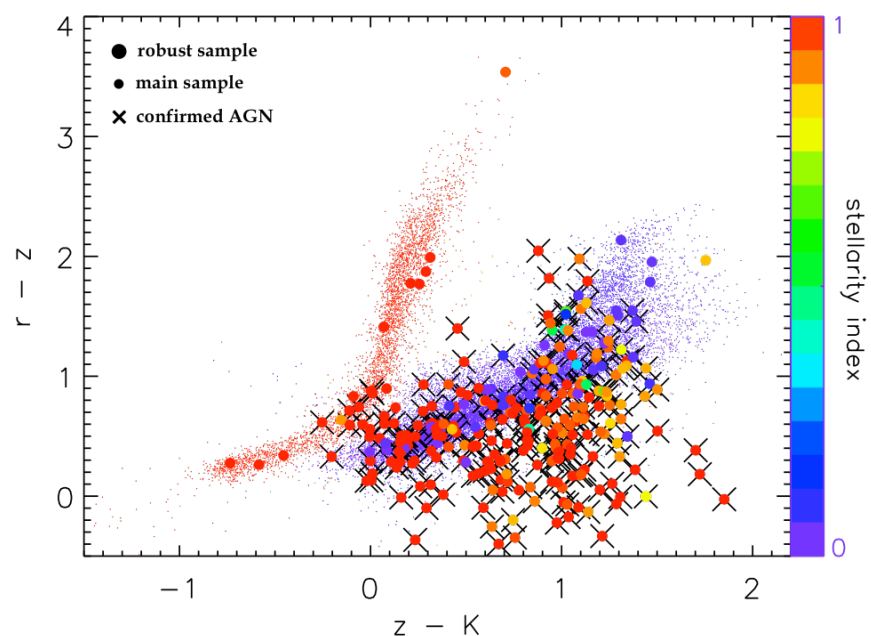

Fig. 6. $r-z$ vs. $z-K$ diagram for 296 out of 299 AGN candidates in the robust sample (large dots) for which $r, z, K$ magnitudes and stellarity index are available. Small dots represent all the objects detected in the VST-COSMOS field for which HST stellarity indices and opti$\mathrm{cal} / \mathrm{NIR}$ color information are available, and are shown as a reference population. Both large and small dots are color-coded according to their stellarity index (right vertical axis). Crosses indicate X-ray emitting variable AGN candidates confirmed by their X-ray properties. It is apparent that the small dots in the plot define two distinct loci: one for stars (red), and one for galaxies (violet). A third class of objects, i.e., quasar-like AGN, is identified from the large dots, corresponding to sources approximately near or below the galaxy locus but mostly with stellarity indices typical of compact sources. Approximately $6 \%$ of the main sample sources shown in Fig. 6 are located in this AGN locus, but are not part of our robust sample of AGN candidates as they are below the variability threshold in the diagram shown in Fig. 3. None of these sources has an X-ray counterpart in the X-ray sample, and $96 \%$ of them are extended sources based on their stellarity. $K_{s}$ magnitudes are taken from McCracken et al. (2010).

extended galaxies. The former form a tight sequence, while the latter tend to occupy a bluer NIR scattered region. Variable sources can change on different scales in different bands, but the effect is minimized by choosing bands close to each other (e.g., Simm et al. 2015). Our $r-z$ versus $z-K$ diagram is shown in Fig. 6. Color information is available for 296 out of 299 sources. When we overplot our variable AGN candidates on this diagram, there is a large overlap with the galaxy region that extends to redder colors, although they are on average more compact than galaxies, as revealed by their stellarity index.

Based on this and on the stellar locus limit introduced by Nakos et al. (2009), we excluded from our robust sample nine stellar sources (3\% of 299 variable AGN candidates) that lie on the stellar sequence far from the galaxy locus. A few additional AGN candidates lie in the overlap region between stars and galaxies, therefore we cannot assess their nature based on this diagram alone. We note that the number of stars detected over our threeyear survey is significantly higher than in our first five-month analysis, where we detected no variables on the stellar locus of the $r-z$ versus $z-K$ diagram, and is also higher than the fraction of stars found in the first 2 square degrees of the VST-CDFS survey that were analyzed in Falocco et al. (2015). This is due to some extent to the longer temporal baseline and the fainter magnitude limit, but mostly to our more relaxed selection criterion (95th percentile threshold, see Sect. 3.3) compared to the one used in our previous works, which allowed lower contamination.

Near-infrared photometry allows us to separate stars from galaxies better than traditional optical colors (e.g., $U-B$ vs.

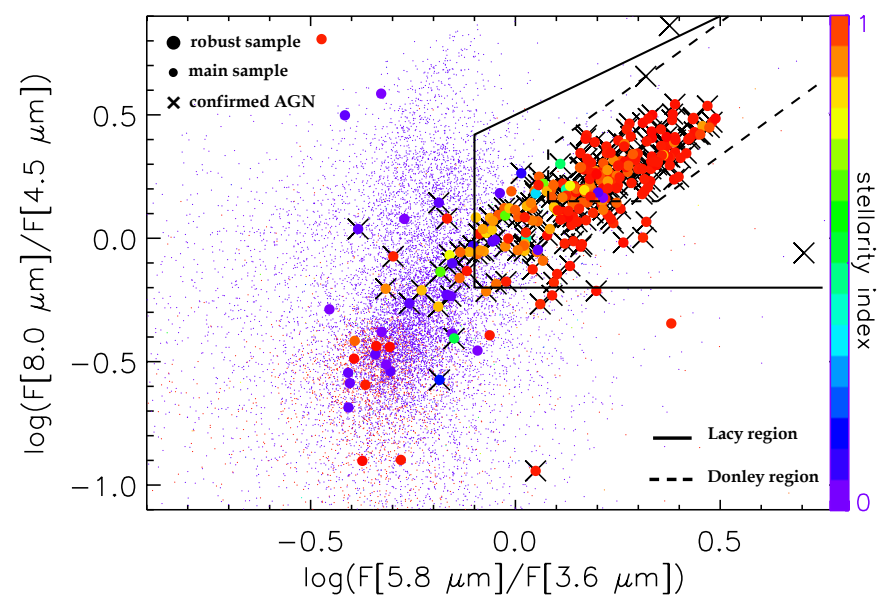

Fig. 7. MIR diagram where colors are obtained as ratios of the fluxes in the four IRAC channels. Symbols are identical to Fig. 6. The solid lines delineate the region where AGN are typically found according to Lacy et al. (2007), while the dashed lines define the less contaminated AGN region identified in Donley et al. (2012). A blob characterized by a high concentration of stars can be seen in the lower left part of the diagram, while inactive galaxies tend to occupy the roughly vertical, scattered sequence defined by small violet dots.

$B-V$ diagrams), and to approximately identify an AGN locus, where point-like sources with colors typical of galaxies are located. Nonetheless, we do see a non-negligible fraction of variable AGN, particularly optically redder and/or fainter AGN, where the host galaxy contamination is more severe or where the nucleus is obscured, whose colors and stellarity are consistent with those of inactive galaxies and which are therefore hard to identify by means of this diagram alone. A caveat here is that this diagnostic is prone to higher contamination than the diagnostics based on X-ray properties because stars, galaxies, and AGN overlap significantly. As a consequence, we only use it to assess the extent of stellar contamination, which corresponds to the $3 \%$ described above.

\subsubsection{Mid-infrared diagnostic}

Lacy et al. (2004) proposed a color-color diagnostic based on the use of mid-infrared (MIR) fluxes $(3.6,4.5,5.8$, and $8.0 \mu \mathrm{m})$ from the Infrared Array Camera (IRAC; e.g., Fazio et al. 2004) of the Spitzer Space Telescope to select AGN. The $8.0 \mu \mathrm{m} / 4.5 \mu \mathrm{m}$ and the $5.8 \mu \mathrm{m} / 3.6 \mu \mathrm{m}$ ratios allow separation of sources whose continuum emission is dominated by different components, such as stellar emission, dust reprocessing in star-forming regions, or nuclear dust heated by the central AGN. In Fig. 7 several distinct loci are visible. Stars and low-redshift passive galaxies are characterized by bluer colors on both axes, and define the denser region that can be observed in the lower left part of the plot. Star-forming galaxies with $z \lesssim 1.5$ are preferentially found on a roughly vertical sequence corresponding to colors with $5.8 \mu \mathrm{m} / 3.6 \mu \mathrm{m}<0.1$ and $8.0 \mu \mathrm{m} / 4.5 \mu \mathrm{m}>0.2$. Finally, quasars and AGN-dominated galaxies define a diagonal locus characterized by red colors on both axes. By defining color criteria, Lacy et al. (2004) delineated boundaries where AGN generally are located on the diagram. This region was slightly modified in Lacy et al. (2007), and we refer to it here and in the following (hereafter, the Lacy region).

As shown by Donley et al. (2012), the Lacy region includes most galaxies with a MIR AGN contribution $\gtrsim 40 \%$, but is not free from contamination by inactive galaxies. A more restrictive 
selection criterion was proposed by Donley et al. (2012), aiming at reducing heavy contamination by star-forming galaxies, which is particularly problematic at high redshift $(z \gtrsim 2)$. Donley et al. (2012) made use of simulations to construct IRAC colors of composite SEDs with different AGN contributions in the redshift range $0-3$, and showed that as the AGN contribution becomes dominant, the IRAC colors of the corresponding sources move onto the locus where sources with perfect IRAC power-law SEDs would lie (hereafter, the Donley region).

Based on the analysis in Falocco et al. (2015), we show our version of the IRAC diagram in Fig. 7, which includes the AGN selection boundaries from both Lacy et al. (2007) and Donley et al. (2012), in order to allow a comparison. IRAC fluxes are from the COSMOS2015 catalog, and are available for 273 out of the 299 sources in our robust sample. We find that $82 \%(223 / 273)$ of the AGN candidates with IRAC fluxes lie within the Lacy region, including two sources confirmed ex novo, that is, not confirmed by any of the previous diagnostics. We caution that this does not constitute a purity estimate because the Lacy region is also known to include non-AGN contaminants. We assess the completeness of our variability selection with respect to MIR diagnostics, recovering 18\% (223/1235) and $55 \%(138 / 249)$ of the sources that fall in the Lacy and Donley region, respectively. However, we note that the Lacy selection is affected by non-AGN contamination and it offers only a very loose lower limit (18\%) on the true completeness, while the Donley selection represent a more robust estimate $(55 \%)$.

We find that variable sources mainly fall on the AGN powerlaw locus described above, which supports the view that a large portion of the sample is comprised of AGN-dominated sources. However, a non-negligible fraction of variability-selected AGN, confirmed by means of other diagnostics, falls outside the Lacy region. In these sources, the MIR host-galaxy emission likely dominates the light contribution from the active nucleus (perhaps due to ongoing star formation), and hence AGN are not easily distinguished from inactive galaxies by means of MIR colors. This confirms that optical variability, when coupled with multiwavelength photometry, can be a powerful tool to identify faint AGN when other color selection methods alone fail.

\section{Discussion}

We investigated the performance of optical variability as an AGN detection method, extending the monitoring baseline from the first five months that were previously studied in De Cicco et al. (2015) up to three years, and the magnitude range to $0.5 \mathrm{mag}$ fainter. The larger baseline, coupled with the wealth of multiwavelength data available in the COSMOS field, allows significant improvements with respect to our previous investigations in COSMOS and the CDFS. In particular, we were able to select a robust sample of 299 AGN candidates on the basis of their optical variability, and employed several multiwavelength diagnostics to confirm and characterize their nature. Table 2 collects and summarizes the results obtained by means of the various diagnostics described in the previous sections, while Table 3 contains a detailed list of all the AGN candidates in our robust sample, with relevant information about them.

Here follows a list of our main findings, together with a comparison with the results from our previous analysis described in De Cicco et al. (2015).

i. We validated as AGN 256 sources in the robust sample, yielding a purity of $86 \%$ (256/299), and demonstrating the effectiveness of optical variability as an AGN selection method. This represents an improvement compared to the $81 \%$ value we
Table 2. Confirmed sources in the robust sample of AGN candidates.

\begin{tabular}{lc}
\hline \hline Confirmed AGN & $\begin{array}{c}256(86 \% \text { of } 299) \\
\text { Confirmed stars }\end{array}$ \\
\hline X/O validation $(\mathrm{X})$ & 248 of 299$)$ \\
Spectroscopic validation (S) & $225(75 \%$ of 299$)$ \\
Lacy region validation $(\mathrm{L})$ & $223(75 \%$ of 299$)$ \\
Donley region validation $(\mathrm{D})$ & $138(46 \%$ of 299$)$ \\
\hline S+X+L+D validation & 134 \\
S+X+L validation & 65 \\
X+L+D validation & 3 \\
S+X validation & 26 \\
X+L validation & 14 \\
L+D validation & 2 \\
Only X validation & 6 \\
Only L validation & 6 \\
\hline Classified sources & 8 \\
with no X-ray counterpart & \\
\hline
\end{tabular}

Notes. We include the number of sources confirmed by individual diagnostics (lines 3-6), as well as the number of sources (when not null) confirmed only by a specific combination of diagnostics, or by a single one of them (lines 7-14).

obtained in De Cicco et al. (2015), particularly considered the adoption of a more relaxed threshold (95\% instead of the $3 \sigma$ used in our previous work) and $0.5 \mathrm{mag}$ fainter detection threshold. In Table 3 we report the percentile each source belongs to; purer samples can be obtained by choosing more restrictive thresholds (e.g., 99th percentile).

Regarding the contamination, our robust sample includes two sources that are spectroscopically classified as stars in the Chandra catalog. These two sources, together with seven additional X-ray undetected sources, lie on the stellar sequence in the diagram shown in Fig. 6. Of the seven stellar candidates with sufficient MIR color information to plot in Fig. 7, six fall within the expected stellar locus. Importantly, none of these sources is classified as AGN by any of the diagnostics we used. As a consequence, we classify all nine of them as stars, implying a stellar contamination of $3 \%$ (9/299). This leaves 34 sources in the robust sample with no classification. In the worst-case scenario where all of them are spurious, the contamination rate would be $14 \%$. The 34 non-confirmed AGN are mostly faint, and only 10 of them have a magnitude $r<23 \mathrm{mag}$.

In Sect. 3.3 we noted that the identification and analysis of $\mathrm{SNe}$ in VST-COSMOS data is currently ongoing (Ragosta et al., in prep.), but at present, there are no identified SNe in our sample of AGN candidatates.

We note that 3 of the 34 sources do not have a counterpart in any of the known COSMOS catalogs, even when we searched for them using a matching radius larger than $1^{\prime \prime}$. These sources were already part of our sample of AGN candidates in De Cicco et al. (2015, see Discussion there); they have average magnitudes $21.9<r<22.4 \mathrm{mag}$, which is well above the HST detection limit. Further investigation is necessary in order to unveil their nature.

We point out that 11 sources belonging to the robust sample lie on the edges of the field, very close to the edge areas that we masked, or very close to other masked regions. Thus they are very likely to be spurious, and we could have excluded them from our robust sample if we had enforced a stricter masking policy. We had noted these sources and their positions at the time we obtained the robust sample, but we chose not to exclude them 

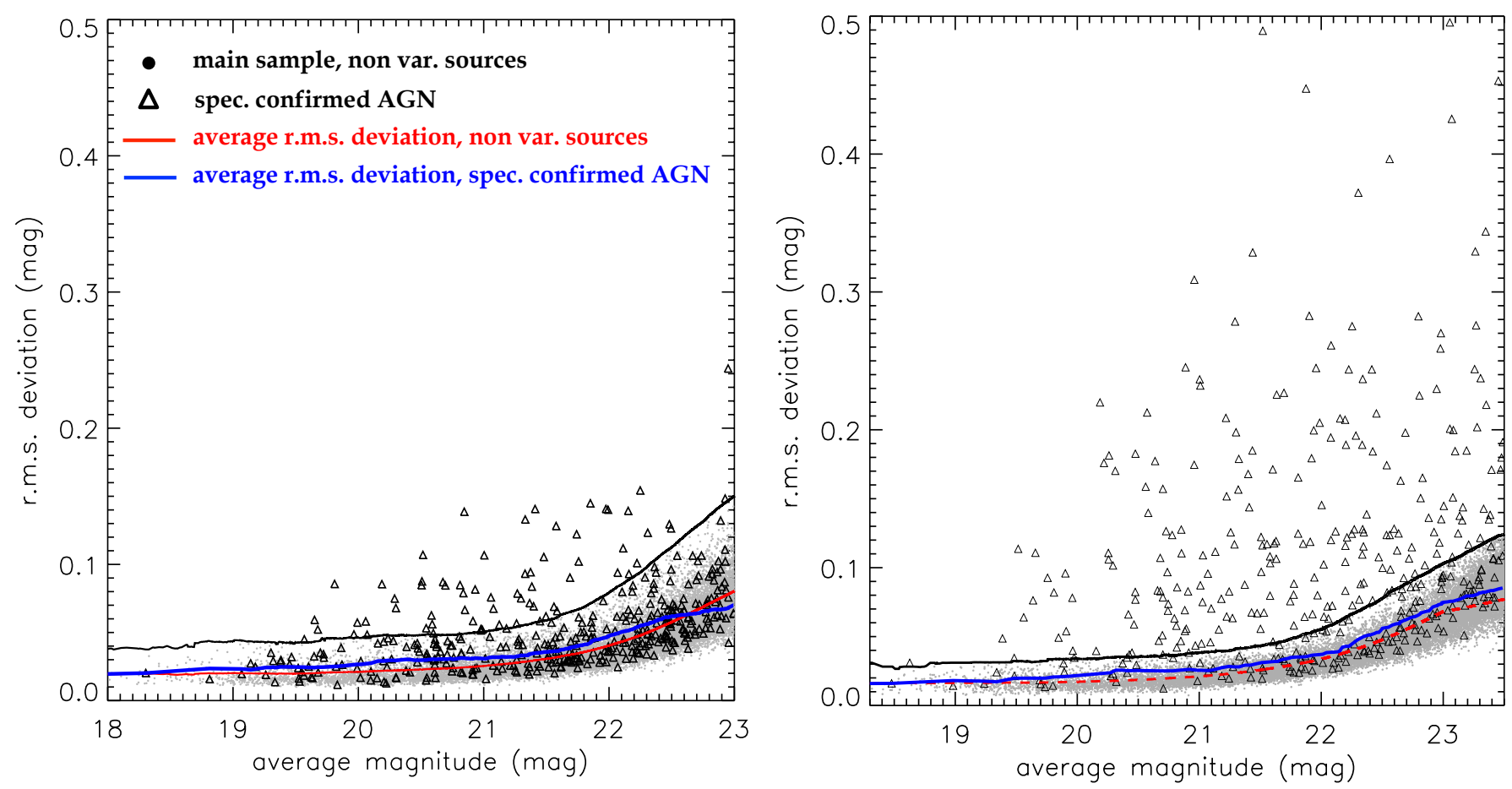

Fig. 8. Light curve r.m.s. as a function of the average magnitude for all the non-variable sources in the main sample (small dots) and for those with an X-ray counterpart and that are spectroscopically confirmed to be AGN (triangles), from our five month analysis (De Cicco et al. 2015, left panel) and the present analysis (right panel). In the left panel the vertical axis has been rescaled to the one in the right panel, while magnitudes are limited to $r(\mathrm{AB}) \leq 23 \mathrm{mag}$. The red and blue curves represent the running average of the r.m.s. deviation for the two subsamples of sources, respectively. In the present work we find 59\% of the sources in the second subsample above the variability threshold (black line), while they were only $15 \%$ in our former analysis.

a priori because we did not wish to introduce a bias in the sample. Excluding them would reduce the robust sample to 288 AGN candidates, yielding a potential confirmation of $89 \%$ and contamination ranging from $3 \%$ to $11 \%$, depending on the nature of the unclassified sources. Three of the remaining 23 sources with no classification based on the used diagnostics are classified as stars in the COSMOS ACS catalog.

When we consider that the analyzed sky area, not including masked regions, corresponds to $\approx 0.83$ square degrees, the number of confirmed AGN returns a density of $\approx 308$ AGN per square degree.

ii. We computed the completeness of AGN in the robust sample with respect to the most reliable sample of AGN available in COSMOS, that is, sources with an X-ray counterpart and a spectroscopic classification as AGN. We obtained $59 \%$ completeness. This percentage is remarkably higher than the $18 \%{ }^{3}$ completeness obtained in De Cicco et al. (2015). This means that our result improved that of De Cicco et al. by a factor of 3.3. In Fig. 8 we show the improvement obtained in this work in the variability detection for X-ray AGN compared to De Cicco et al. (2015) by reporting two diagrams showing the completeness for both our previous and current analyses. In the five-month analysis (left panel) VST sources with an X-ray counterpart on average exhibited a higher variability than the whole sample of VST sources, although $>80 \%$ of the sources with an X-ray counterpart fell below

3 De Cicco et al. (2015) computed the completeness with respect to AGN confirmed by spectroscopic and/or X-ray properties. Here we limited ourselves to spectroscopy and did not take X-ray properties into account. This explains why we reported a completeness of $15 \%$ in 2015 instead of the $18 \%$ we report here. our variability threshold. At the time we predicted, based on the red-noise variability typical of AGN, that a longer baseline would have returned a larger sample of sources above the variability threshold, and hence a much higher completeness (see Discussion and Fig. 8 in De Cicco et al. 2015); this is indeed what we found in the current analysis (right panel), where the completeness rises to $59 \%$ and the fraction of sources below the threshold hence drops from $>80 \%$ to $41 \%$.

The completeness for unobscured AGN is now $82 \%$, and it is $18 \%$ for obscured AGN. De Cicco et al. (2015) reported a $25 \%$ and $6 \%$ completeness, respectively. We note that our past analysis was limited to sources with $r(\mathrm{AB}) \leq 23 \mathrm{mag}$, while here we go half a magnitude deeper. Anyway, we verified that the results are not affected by the different magnitude threshold, and would be the same if we adopted the same cut. This suggests that the improvement in the detection rate is an effect of the longer baseline, and Fig. 8 confirms that.

The fraction of unobscured AGN we retrieve is eight times larger than that of obscured AGN. This is expected because optical variability is biased toward the first class of AGN: we observe their inner regions directly (e.g., Padovani et al. 2017, and references therein).

Taking the cue from what we did in De Cicco et al. (2015), we also computed the completeness in four magnitude bins, from $r=20$ to $23.5 \mathrm{mag}$. We obtain $80 \%, 66 \%$, and $53 \%$, respectively, for the first three bins, which have a size of one magnitude. Similarly to De Cicco et al. (2015), these fractions are higher for brighter sources. The corresponding values in our previous work are $26 \%, 23 \%$, and $5 \%$. These fractions cannot properly be compared with those reported in De Cicco et al. (2015) because 


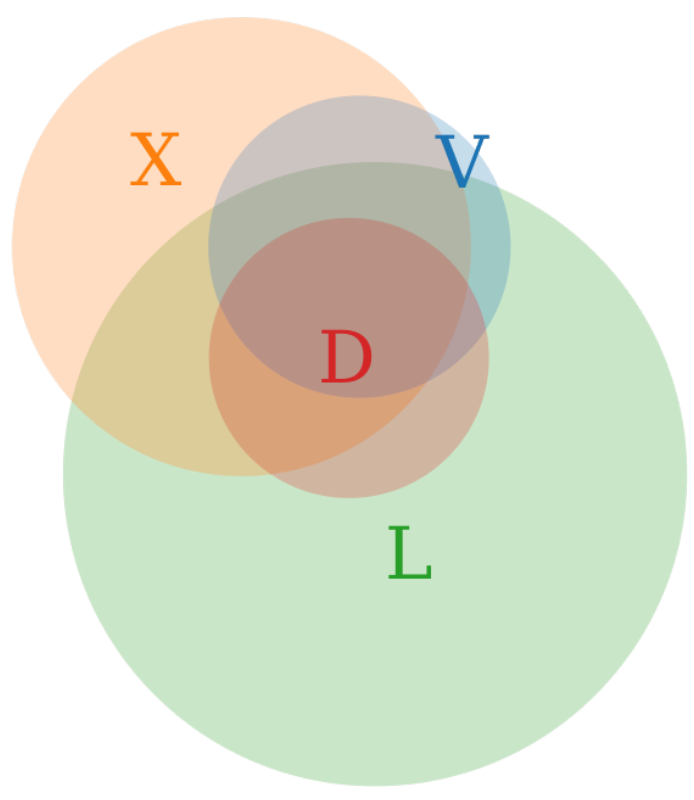

Fig. 9. Venn diagram combining the samples of AGN selected by means of different diagnostics: optical variability $(\mathrm{V}), \mathrm{X}$-ray properties $(\mathrm{X}$, from the $\mathrm{X} / \mathrm{O}$ diagram), Lacy region (L), and Donley region (D). The circle sizes, as well as the overlap sizes, are quantitatively correct. The sample of optically variable sources includes the 34 sources that are not confirmed by any other diagnostics, while it does not include the 9 sources that are confirmed as stars (see main text). It is apparent that the variability-selected sample largely overlaps the X-ray selected and the Lacy-selected AGN, while the overlap with the Donley-selected sample is only partial.

the definition we adopted here for the completeness is stricter. The large difference between the values reported for each bin is clearly dependent on the longer baseline available to us here, as we noted above. The remaining bin has the size of half a magnitude and has no correspondence in our previous analysis. The completeness in this case is $45 \%$.

In Sect. 4.1 we reported that the completeness with respect to the AGN confirmed by the X/O diagram is $37 \%$. We note that the $\mathrm{X}$ ray sample includes both unobscured and obscured AGN, and that AGN selection based on optical variability is biased against the latter class. This is a possible explanation for the $37 \%$ completeness that we obtain.

iii. In Sect. 3.3 we stated that our robust sample does not include 93 sources flagged 3 because of their doubtful variability. Based on the diagnostics we used throughout this work, we would confirm 12 of them as AGN. This number includes 3 sources that are confirmed only by the Lacy diagnostic, which, as we described in Sect. 4.3.2, is affected by non-AGN contamination. As a consequence, the number of sources labeled 3 with a reliable confirmation of their AGN nature is 9/93 (10\%), indicating that we did not bias our results when we removed sources that are affected by aesthetic or photometric problems.

In Fig. 9 we show a Venn diagram that summarizes the main results we obtained by the various diagnostics we used to validate our sources. The sample selected by means of optical variability largely overlaps the X-ray and IR samples, but is partly complementary to them. This is due to our relatively bright limit and the approach adopted in this work, which selects bright quasar-like AGN. Other works show that addressing the problem of the host galaxy contamination through higher resolution instruments (e.g., Villforth et al. 2010; Sarajedini et al. 2011; Pouliasis et al. 2016, 2019) or through image subtraction

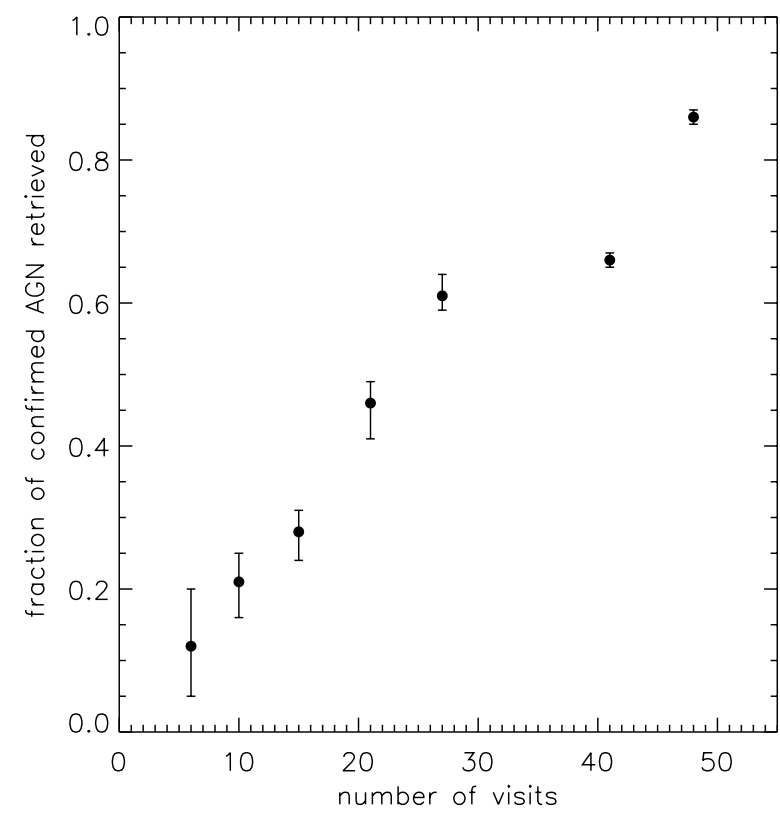

Fig. 10. Fraction of confirmed AGN that are retrieved by selecting an increasing number of visits over a fixed baseline of 3.3 years, which corresponds to the baseline covered in this work, and is the longest sampled timescale for VST-COSMOS data to date. Each point is the average result of 10 random simulations, the error bars being the corresponding thresholds for the 10th and the 90th percentiles. The plot shows that as the number of visits increases, we are able to retrieve a larger fraction of AGN. A denser sampling leads to a reduction in the size of the error bars, as an effect of the reduced differences among the possible patterns that can be chosen for the simulation.

techniques (e.g., Botticella et al. 2017) allows recovering complementary samples of low-luminosity or X-ray faint AGN. In any case, variability allows us to confirm AGN candidates identified by means of less robust or more contaminated diagnostics (e.g., Lacy et al. 2007).

The 256 confirmed AGN were found by analyzing data from 54 visits over a three-year baseline. In order to assess how the sampling cadence could affect the AGN detection efficiency of future surveys, we made some tests by varying the number of visits over a fixed baseline of 3.3 years. When possible, we included visits from each of the three seasons, in order to obtain a coverage as homogeneous as possible. Consistent with what we did in this work (see Sect. 3), we always required sources to be detected in at least half the visits. We show the results of this test in Fig. 10. The test shows how significantly a denser sampling affects the detection efficiency. This effect is often underestimated when monitoring campaigns are planned because it is incorrectly assumed that just increasing the baseline is sufficient to increase the number of detections, even if this means dramatically reducing the sampled cadence.

Sections 1 and 3 highlighted that this work extends the analysis of De Cicco et al. (2015) to a longer baseline, and uses a different approach to correct magnitudes (reference visit vs. growth curves), to select the sample of sources to include in the analysis (detection required in at least $50 \%$ vs. $20 \%$ of the visits), and to define a variability threshold (95th percentile vs. $3 \sigma$ threshold). This work also makes use of a sigma-clipping algorithm when $\left\langle\mathrm{mag}^{l c}\right\rangle$ and $\sigma^{l c}$ are computed from the light curve of each source. It is thus worth comparing briefly the results obtained in our previous work with those we would obtain if we were to use this new approach in the analysis of the same dataset as in 
De Cicco et al. (2015), which consisted of 27 visits over the first season of the VST survey of COSMOS. We note that visit 27 is one of those excluded from the present work (see Sect. 3.1 and Fig. 2). For the sake of consistency, we included this visit in the analysis performed to compare the results obtained with the two approaches over the same season. For the same reason, the sample of sources is now cut to $r \leq 23 \mathrm{mag}$, as in De Cicco et al. (2015).

De Cicco et al. (2015) obtained a sample of 83 AGN candidates (hereafter, sample 1), and confirmed 67 as AGN (81\%). Following the new approach outlined in this work, we would obtain a sample of 129 AGN candidates (hereafter, sample 2), and confirm 101 as AGN (78\%), thus obtaining consistent results. In sample 2 we recover $84 \%$ of the AGN candidates and $90 \%$ of the confirmed AGN in sample 1. The constraint on the number of detections does not significantly affect the result because most of the sources in each of the inspected samples were detected in almost all the visits. We expect sample 2 to be larger than sample 1 due to the different threshold adopted, which now roughly corresponds to a $1.5 \sigma$ threshold, rather than $3 \sigma$. The sigma-clipping algorithm, on the other hand, reduces the inclusion of spurious sources in the sample of AGN candidates.

There is one major factor to take into account, which is the red-noise type variability of AGN. The complete analysis over the baseline of 3.3 years returns $99 \%$ of the confirmed AGN from sample 1 that do not fall in areas that in the new analysis we chose to mask. It also shows that there is only one AGN confirmed in sample 1 that is not retrieved in the robust sample because it is below the variability threshold. All this proves that the adopted approach, coupled with the longer baseline, leads to improved results with respect to De Cicco et al. (2015), as is also shown by the higher values obtained for purity and completeness.

In Sect. 2 we noted that so far, our work has focused on $r$-band data. A complementary analysis of COSMOS $g$ - and $i$-band variability, together with a multiband analysis combining $g, r$, and $i$ data, will be presented in a forthcoming paper. This will offer an opportunity to investigate correlated variability in different bands, together with the dependence of AGN variability on the specific wavelength range, and also average and time-dependent color selection (e.g., Richards et al. 2015). The multiband analysis will also retrieve more robust samples of candidates, simultaneously varying in multiple bands and therefore minimizing contributions from contaminants.

Acknowledgements. We acknowledge support from CONICYT grants BasalCATA Basal AFB-170002 (D.D., F.E.B.), the Ministry of Economy, Development, and Tourism's Millennium Science Initiative through grant IC120009, awarded to The Millennium Institute of Astrophysics, MAS (D.D., F.E.B., G.P.), the ASI-INAF agreement n.2017-14-H.0.s (M.P., F.V., G.C.), the Italian Ministry of Foreign Affairs and International Cooperation (MAECI Grant Number ZA18GR02) and the South African Department of Science and Technology's National Research Foundation (DST-NRF Grant Number 113121) as part of the ISARP RADIOSKY2020 Joint Research Scheme (M.V.).

\section{References}

Alexander, D. M., Vignali, C., Bauer, F. E., et al. 2002, AJ, 123, 1149 Bertin, E., \& Arnouts, S. 1996, A\&AS, 117, 393

Botticella, M. T., Cappellaro, E., Pignata, G., et al. 2013, The Messenger, 151, 29

Botticella, M. T., Cappellaro, E., Greggio, L., et al. 2017, A\&A, 598, A50 Boutsia, K., Leibundgut, B., Trevese, D., \& Vagnetti, F. 2009, A\&A, 497, 81 Brandt, W. N., \& Alexander, D. M. 2015, A\&ARv, 23, 1 Brandt, W. N., \& Hasinger, G. 2005, ARA\&A, 43, 827

Brandt, W. N., Ni, Q., Yang, G., et al. 2018, ArXiv e-prints [arXiv:1811.06542]

Brusa, M., Civano, F., Comastri, A., et al. 2010, ApJ, 716, 348

Capaccioli, M., \& Schipani, P. 2011, The Messenger, 146, 2

Capaccioli, M., Spavone, M., Grado, A., et al. 2015, A\&A, 581, A10

Capak, P., Aussel, H., Ajiki, M., et al. 2007, ApJS, 172, 99

Chiaraluce, E., Vagnetti, F., Tombesi, F., \& Paolillo, M. 2018, A\&A, 619, A95

Choi, Y., Gibson, R. R., Becker, A. C., et al. 2014, ApJ, 782, 37

Civano, F., Elvis, M., Brusa, M., et al. 2012, ApJS, 201, 30

Civano, F., Marchesi, S., Comastri, A., et al. 2016, ApJ, 819, 62

De Cicco, D., Paolillo, M., Covone, G., et al. 2015, A\&A, 574, A112

Donley, J. L., Koekemoer, A. M., Brusa, M., et al. 2012, ApJ, 748, 142

Falocco, S., Paolillo, M., Covone, G., et al. 2015, A\&A, 579, A115

Fazio, G. G., Hora, J. L., Allen, L. E., et al. 2004, ApJS, 154, 10

Ferrarese, L., \& Merritt, D. 2000, ApJ, 539, L9

Gaskell, C. M., \& Klimek, E. S. 2003, Astron. Astrophys. Trans., 22, 661

Grado, A., Capaccioli, M., Limatola, L., \& Getman, F. 2012, Mem. Soc. Astron. It. Suppl., 19, 362

Graham, M. J., Djorgovski, S. G., Drake, A. J., et al. 2014, MNRAS, 439, 703

Huang, Z., Radovich, M., Grado, A., et al. 2011, A\&A, 529, A93

Klesman, A., \& Sarajedini, V. 2007, ApJ, 665, 225

Koekemoer, A. M., Aussel, H., Calzetti, D., et al. 2007, ApJS, 172, 196

Kuijken, K. 2011, The Messenger, 146, 8

Lacy, M., Storrie-Lombardi, L. J., Sajina, A., et al. 2004, ApJS, 154, 166

Lacy, M., Petric, A. O., Sajina, A., et al. 2007, AJ, 133, 186

Laigle, C., McCracken, H. J., Ilbert, O., et al. 2016, ApJS, 224, 24

Lintott, C. J., Schawinski, K., Keel, W., et al. 2009, MNRAS, 399, 129

LSST Science Collaboration (Abell, P. A., et al.) 2009, ArXiv e-prints [arXiv:0912.0201]

Maccacaro, T., Gioia, I. M., Wolter, A., Zamorani, G., \& Stocke, J. T. 1988, ApJ, 326,680

MacLeod, C. L., Brooks, K., Ivezić, Ž., et al. 2011, ApJ, 728, 26

Magorrian, J., Tremaine, S., Richstone, D., et al. 1998, AJ, 115, 2285

Mainieri, V., Bergeron, J., Hasinger, G., et al. 2002, A\&A, 393, 425

Marchesi, S., Civano, F., Elvis, M., et al. 2016, ApJ, 817, 34

McCracken, H. J., Capak, P., Salvato, M., et al. 2010, ApJ, 708, 202

Mushotzky, R. 2004, in Supermassive Black Holes in the Distant Universe, ed.

A. J. Barger, Astrophys. Space Sci. Lib., 308, 53

Nakos, T., Willis, J. P., Andreon, S., et al. 2009, A\&A, 494, 579

Padovani, P., Alexander, D. M., Assef, R. J., et al. 2017, A\&ARv, 25, 2

Paolillo, M., Papadakis, I., Brandt, W. N., et al. 2017, MNRAS, 471, 4398

Pouliasis, E., Georgantopoulos, I., Bonanos, A., \& HCV Team 2016, in Active Galactic Nuclei: What's in a Name?, 95

Pouliasis, E., Georgantopoulos, I., Bonanos, A. Z., et al. 2019, MNRAS, 487, 4285

Richards, G. T., Myers, A. D., Peters, C. M., et al. 2015, ApJS, 219, 39

Salvato, M., Hasinger, G., Ilbert, O., et al. 2009, ApJ, 690, 1250

Salvato, M., Buchner, J., Budavári, T., et al. 2018, MNRAS, 473, 4937

Sarajedini, V. L., Koo, D. C., Klesman, A. J., et al. 2011, ApJ, 731, 97

Schmidt, K. B., Marshall, P. J., Rix, H.-W., et al. 2010, ApJ, 714, 1194

Scoville, N., Abraham, R. G., Aussel, H., et al. 2007, ApJS, 172, 38

Simm, T., Saglia, R., Salvato, M., et al. 2015, A\&A, 584, A106

Trevese, D., Boutsia, K., Vagnetti, F., Cappellaro, E., \& Puccetti, S. 2008, A\&A, 488, 73

Ulrich, M.-H., Maraschi, L., \& Urry, C. M. 1997, ARA\&A, 35, 445

Villforth, C., Koekemoer, A. M., \& Grogin, N. A. 2010, ApJ, 723, 737

Xue, Y. Q., Luo, B., Brandt, W. N., et al. 2011, ApJS, 195, 10 